\title{
Alterations in cyclin D1 and cyclin-dependent kinase 4 expression in the amygdalae of post-traumatic stress disorder rats
}

\author{
MING CONG ${ }^{1,2}$, LILI WEN $^{1}$, FANG HAN $^{1}$, YANHAO XU $^{1}$ and YUXIU SHI ${ }^{1}$ \\ ${ }^{1}$ PTSD Laboratory, Department of Histology and Embryology, China Medical University, \\ Shenyang, Liaoning 110122; ${ }^{2}$ Department of Neurosurgery, First Affiliated Hospital \\ of Jinzhou Medical University, Jinzhou, Liaoning 121001, P.R. China
}

Received April 7, 2017; Accepted September 8, 2017

DOI: $10.3892 / \mathrm{mmr} .2017 .7613$

\begin{abstract}
The amygdalae are an important component of the human limbic system and exhibit a key role in emotional and behavioral reactions. Previous studies have demonstrated abnormal function and morphology in the amygdalae of post-traumatic stress disorder (PTSD)-like animal models, however the underlying molecular mechanisms remain elusive. The authors have previously demonstrated that PTSD induced increased apoptosis in the amygdala of PTSD-like animals. Cyclin D1 and cyclin-dependent kinase 4 (CDK4) are two important regulators of the cell cycle. The study explored the expression of cyclin D1 and CDK4 in the amygdala in PTSD. The single-prolonged stress (SPS) rat model was used as a PTSD-like model. Ultrastructural alterations of cells in the amygdala were observed using transmission electron microscopy (TEM). 4',6-Diamidino-2-phenylindole (DAPI) fluorescence was employed to detect nuclear pycnosis. Cyclin D1 and CDK4 expression in the amygdala cells was examined using immunofluorescence, Western blotting and reverse transcription-quantitative polymerase chain reaction. TEM revealed morphological alterations to the amygdala cells of the SPS rats. DAPI-stained nuclear brightness levels differed between the control and SPS groups. Expression of cyclin D1 and CDK4 in the amygdala increased gradually 1 day and 4 days following SPS stimulation, and peaked 7 days following SPS stimulation at the protein and mRNA levels, in comparison with the control rats. These findings suggest that SPS resulted in increased cyclin D1 and CDK4 expression, which may accelerate cell apoptosis. This may be associated with SPS-induced abnormal function and structure of the amygdala.
\end{abstract}

Correspondence to: Professor Yuxiu Shi or Professor Yanhao Xu, PTSD Laboratory, Department of Histology and Embryology, China Medical University, 77 Puhe Road, Shenyang North New Area, Shenyang, Liaoning 110122, P.R. China

E-mail: shiyuxiu17@163.com

E-mail: xyh@cast.org.cn

Key words: post-traumatic stress disorder, cell apoptosis, cell cycle, cyclin D1, cyclin-dependent protein kinases

\section{Introduction}

Post-traumatic stress disorder (PTSD) is a common condition characterized by persistent mental disturbances following a traumatic event. The incidence of PTSD has gradually increased, as a result of frequent natural disasters such as earthquakes and tsunamis and incidents of violence such as war, terrorist attacks. and serious traffic accidents. It has been previously demonstrated that extremely severe psychological trauma contributes considerably to this disorder (1).

The main symptoms of PTSD include uncontrollable re-experiencing of the trauma, escape behavior, and irritability caused by hyperarousal (1). Deep memories of events or situations that evoke intense fear in PTSD patients may be related to over-activity of parts of the limbic system, specifically the amygdala and hippocampus (2). The amygdala is an important component of the human limbic system, responsible for emotional and behavioral reactions and is considered to play a critical role in panic reactions, particularly in terror symptoms (3-5). The amygdala can be divided into three distinct subregions: The central nucleus, the corticomedial nucleus, and the basolateral nucleus (6), the largest of these three. It is the key region for fear initiation and has been the focus of significant attention. Vyas and co-workers reported that chronic, unpredictable stress induces atrophy in bipolar neurons of the basolateral amygdalae (7). We focus here on observing changes of the basolateral nucleus.

Studies have revealed that the number of pyramidal neuron dendrites of PTSD-like rats declined in the hippocampus, but increased in the amygdalae (8). Other studies have found that the amygdala is oversensitive in PTSD, and its degree of activation is in direct proportion to severity of PTSD symptoms (9). This research clearly illustrates that changes in the amygdala play a significant role in the morbidity of PTSD (10). The results demonstrated that the amygdalae are consistently hyperactivated bilaterally in PTSD patients (11). Kühn did not find amygdala volume differences (12), but other studies have found that the volume of the amygdalae of PTSD patients shrink, and the degree of volume change correlates positively with the severity of the PTSD symptoms $(13,14)$.

One of the main mechanisms for maintenance of tissue homeostasis is the control and regulation of apoptosis (15). Evidence suggests that the reduction of volume in the 
amygdala of PTSD patients may be related to nerve cell apoptosis (16), and the death and loss of neurons in the amygdala is a generally observed phenomenon in PTSD (17). However, the molecular mechanisms underlying this phenomenon remain elusive. Single-prolonged stress (SPS) is one of the animal models proposed for PTSD (18). The SPS rat shows enhanced inhibition of the hypothalamo-pituitary-adrenal (HPA) axis, which has been frequently demonstrated in patients with PTSD. It has been reported that SPS induces apoptosis of the hippocampus and amygdala in the rat model by regulating $\mathrm{Ca}^{2+}$-calmodulin $(\mathrm{CaM})-\mathrm{Ca}^{2+} / \mathrm{CaM}$-dependent protein kinase II (CaMKII) signaling pathways (19-21). Our previous studies have demonstrated that apoptosis of neurons is mainly carried out via mitochondria-related apoptosis factors, such as caspase-9, caspase-3 and cytochrome c, but the genes Bcl-2 and Bax also play a significant role $(22,23)$.

The cell cycle refers to the process in which eukaryotic cells carry out cell proliferation through mitosis. It consists of four phases: Pre-DNA synthesis (G1), DNA synthesis (S), post-DNA synthesis (G2), and the mitotic phase (M). The cell cycle is complicated and delicate, requiring the participation of multiple protein regulatory factors (24). These regulatory factors include cyclins, cyclin-dependent kinases (CDKs), and CDK inhibitor proteins (CDKIs). During the process, cyclins and CDKs may form specific compounds, which may further act on the specific substrates, and carry out accurate regulation of the cell cycle from beginning to end. Cyclin is the regulatory molecule, whereas CDK is the catalytic subunit (25). Research has found that there are corresponding cyclins and CDKs in each stage of the cell cycle (26). CDK4 protein is expressed substantially in the post-G1 phase, and combines with the cyclin D1 protein, which may finally promote the differentiation of cells from G1 to $S$ phase (27) and begin the process of mitosis.

CDK4 and cyclin D1 are important cell cycle regulators of the G1-S transition (28). Mature neural cells are the end cell type formed after the differentiation of neuroepithelial stem cells and have already lost the capacity for division and multiplication. Deregulation of the cell cycle is associated with injury to the central nervous system. When injured, neural cells maintain their reactivation potential and upregulate signaling molecules responsible for controlling the cell cycle, including cyclin D1 and CDK4 $(29,30)$. However, reactivation of the cell cycle is disorganized and cannot control the complete division and proliferation of cells. Consequently, the cell cycle stops at a certain phase, arresting the cell cycle arrest and giving rise to apoptosis $(31,32)$. Studies have shown that Cyclin D1 and CDK4 present excessive expression of the neural cell in ischemic brain injury, and this may give rise to neural cell apoptosis $(33,34)$.

This study describes further investigation into whether SPS affects the expression of cyclin D1 and CDK4 in the neural cells of the amygdala, and whether this alteration is correlated with the progression of PTSD. Our results may provide new insight for the treatment and prevention of PTSD.

\section{Materials and methods}

Experimental animals and grouping. The animal experiments were approved by the Institutional Animal Care and Use
Committee of China Medical University. Male Wistar rats weighing 130 to $170 \mathrm{~g}$ and aged 8 to 10 weeks were subjected to a PTSD model using the SPS method. During the experiment, the rats were housed in groups and were maintained in a $12 / 12 \mathrm{~h} \mathrm{light/dark} \mathrm{cycle,} \mathrm{at} \mathrm{environmental} \mathrm{temperatures}$ of $22-25^{\circ} \mathrm{C}$, and fed and watered ad libitum. The rats $(\mathrm{n}=100)$ were randomly divided into five groups of 20 rats per group: A control group, SPS 1-day group (1 day after SPS exposure), SPS 4-day group (4 days after SPS exposure), SPS 7-day group (7 days after SPS exposure), and SPS 14-day group (14 days after SPS exposure). In each group of 20 rats, 5 were used for western blotting, 5 for immunofluorescence studies, 5 for quantitative polymerase chain reaction (qPCR), and 5 for transmission electron microscopy (TEM). All experimental procedures were approved by the Ethics Committee of China Medical University and conducted in accordance with the Guidelines Principles on Animal Experimentation for Laboratory Animal Science, China Medical University.

Modeling of PTSD rat: SPS. SPS is recognized internationally as a rat model for PTSD research, and is described in the following steps (35). Rats were immobilized for $2 \mathrm{~h}$, followed immediately by a 20 -min forced swim conducted in an acrylic cylindrical tank filled with water $\left(40 \mathrm{~cm}\right.$ height, $25^{\circ} \mathrm{C}$ water temperature). After recuperating for $15 \mathrm{~min}$, rats were placed in a shock chamber and anesthetized with ether, and then returned to their home cages without any stimulation, and fed regularly until sampling.

TEM. Following SPS, the rats were anesthetized with ether and $2 \%$ pentobarbital. The rats were transcardially perfused with $250 \mathrm{ml}$ cold phosphate-buffered saline (PBS) to remove intravascular blood. Amygdala tissues were sampled and fixed with $2.5 \%$ glutaraldehyde. The tissue samples were processed for regular TEM, in semi-thin sections by standard techniques. The $70 \mathrm{~nm}$ sections were positioned for observation and imaging under a transmission electron microscope (JEOL-1200EX; JEOL, Tokyo, Japan).

Tissue preparation. Rats were deeply anesthetized, then perfused with cold PBS and 4\% paraformaldehyde (PFA). Amygdala tissue was postfixed in $4 \%$ PFA for $3 \mathrm{~h}$, and then immersed in Holt's liquid (30\% sucrose, $0.01 \mathrm{~mol} / 1 \mathrm{PBS}$ preparation) until it reached the bottom. Coronal sections were cut at a thickness of $10 \mu \mathrm{m}$ using a cryostat vibratome, and this cryopreservation used for the immunofluorescence staining.

Immunofluorescence double staining. The section was dried for $2 \mathrm{~h}$ and rinsed with PBS three times for $5 \mathrm{~min}$, washed for 10 min with $0.3 \%$ Triton liquid, and dried with absorbent paper to remove the Triton liquid. Sections were blocked in $5 \%$ bovine serum albumin in PBS at room temperature for $20 \mathrm{~min}$, and incubated with a primary antibody against CDK4 (1:200 in PBS) (ImmunoWay Biotechnology Co., Newark, DE, USA) overnight at $4^{\circ} \mathrm{C}$, with PBS used as a negative control. Following three washes in PBS, sections were incubated with anti-rabbit IgG (1:50) labeled with Cy3 (Nanjing KeyGen Biotech. Co., Ltd., Nanjing, China) and anti-rabbit IgG (1:50) labeled with 4',6-diamidino-2-phenylindole (DAPI; Nanjing KeyGen Biotech. Co., Ltd.), respectively, and maintained in 
darkness overnight at $4^{\circ} \mathrm{C}$. They were then rinsed with PBS liquid three times for $5 \mathrm{~min}$, and closed with glycerinum. Images were acquired using a Nikon laser scanning confocal microscope with EZ-C1 3.70 FreeViewer image analysis software (Nikon, Tokyo, Japan). The positive cell fluorescence strength of CDK4 and the positive nucleus fluorescence strength of DAPI staining of the 5 groups were measured. Fluorescence strength was indicated as $\mathrm{AU} / \mu \mathrm{m}^{2}$. Five slides were randomly selected from each group; in each, 5 visual fields (x40) in the amygdala were randomly selected. For every animal, approximately 100 cells were counted.

Detection of cyclin D1 and CDK4 with western blotting. Rats were deeply anesthetized, intracardially perfused with cold PBS, and the amygdala tissue of the controls and the SPS group at each time point was rapidly removed. Then the basolateral amygdalae were dissected according to the atlas of Paxinos and Watson (36). The tissue was homogenized in lysis buffer containing protease inhibitors with further sonication and centrifugation at $12,000 \mathrm{rev} / \mathrm{min}$ for $20 \mathrm{~min}$. Total protein content of the supernatant was quantified using a bicinchoninic acid (BCA) assay kit. An amount of $50 \mu \mathrm{g}$ of the total protein was electrophoresed in $10 \%$ sodium dodecyl sulfate-polyacrylamide gel electrophoresis (SDS-PAGE) gel and transferred to a polyvinylidene fluoride (PVDF) membrane. Monoclonal antibody cyclin D1 (sc-735; Santa Cruz Biotechnology, Inc., Santa Cruz, CA, USA) at 1:600 dilution and rabbit polyclonal antibody CDK4 (sc-260; Santa Cruz Biotechnology, Inc.) also at 1:600 dilution were used as primary antibodies. Following three washes in Tris-buffered saline with Tween-20, the membranes were incubated with $\mathrm{IgG}$ labeled with horseradish peroxidase (HRP). The HRP-IgG antibody (Hebei Bio-High Technology Development Co., Shijiazhuang, China) exposure $(1: 1,000)$ continued for $2 \mathrm{~h}$ at room temperature. Immunoreactivity was visualized with an enhanced chemiluminescence reagent kit. The integrated density value was calculated with a Fluorchem V 2.0 system and normalized to the values for glyceraldehyde 3-phosphate dehydrogenase (GAPDH; Wuhan Boster Biological. Technology, Ltd., Wuhan, China).

Detection of cyclin D1 mRNA, and CDK4 mRNA using reverse transcription $(R T)-q P C R$. After anesthesia with $2 \%$ pentobarbital, amygdala tissue was isolated from the cerebral hemisphere of rates in the control group and the SPS group. Total RNA was isolated from the amygdala tissue by Trizol assay. First-strand cDNA synthesis and amplification were performed according to a kit from Takara Bio, Inc. (Otsu, Japan). An amount of $2 \mu \mathrm{l}$ cDNA was removed for PCR amplification; the amplification reaction system is shown in Table I and the primer sequences are shown in Table II (Shenggong Biotech Co., Shanghai, China). Amplification conditions were as follows: Denaturing at $95^{\circ} \mathrm{C}$ for $30 \mathrm{sec}$; amplification at $95^{\circ} \mathrm{C}$ for $5 \mathrm{sec}$, and heating at $60^{\circ} \mathrm{C}$ for $20 \mathrm{sec}$. Forty cycles were performed with the solution at a temperature of $95^{\circ} \mathrm{C}$ for $10 \mathrm{sec}$, and $65^{\circ} \mathrm{C}$ for $10 \mathrm{sec}$, and cooled $40^{\circ} \mathrm{C}$ for $30 \mathrm{sec}$.

All quantifications were normalized to an endogenous GAPDH control. Cyclin D1 mRNA and CDK4 mRNA expression levels were validated by using the Real-Time fluorescence qPCR instrument LightCycler 480, according to the manufacturer's instructions. After the end qPCR amplification, $\mathrm{Cq}$
Table I. Amplification reaction system.

\begin{tabular}{lc}
\hline Reagent & Volume \\
\hline SYBR & $10.0 \mu 1$ \\
PCR forward primer & $0.4 \mu 1$ \\
PCR reverse primer & $0.4 \mu 1$ \\
cDNA & $2.0 \mu 1$ \\
$\mathrm{dH}_{2} \mathrm{O}$ & $7.2 \mu 1$ \\
Total & $20.0 \mu 1$
\end{tabular}

value of the cyclin D1/CDK4 and GAPDH in control group and the tested group were outputted. Normalize the $\mathrm{Cq}$ value of the target gene with the $\mathrm{Cq}$ value of the GAPDH gene with the formula: $\triangle \mathrm{Cq}=\mathrm{Cq}$ (Goal)-Cq (GAPDH). And then normalize the $\Delta \mathrm{Cq}$ value of the test group with the $\Delta \mathrm{CT}$ value of the control group with $\Delta \Delta \mathrm{Cq}=\Delta \mathrm{Cq}$ (tested group) $-\Delta \mathrm{Cq}$ (control group). Next, calculate the ratio of expression levels of the tested group which is relative to the control group with $2^{-\Delta \Delta \mathrm{Cq}}$. Finally, input the results of $2^{-\Delta \Delta \mathrm{Cq}}$ of all groups into the spss statistical software to draw the chart. The relative expression level between treatments was standardization of the target mRNA. We repeated the experiment three times and obtained similar results.

Statistical analysis. All of the results were analyzed with SPSS 18.0 statistical software (SPSS, Inc., Chicago, IL, USA), and data were presented as mean \pm SEM. Multiple comparisons were carried out using one-way analysis of variance followed by the Tukey post hoc test; results of the two groups were compared with the Student's t-test. $\mathrm{P}<0.05$ was considered to indicate a statistically significant difference.

\section{Results}

Observation of morphological changes of amygdala cells with TEM. In the amygdalae of the control group, evenly distributed chromatin in the nuclear and complete nuclear membrane was observed (Fig. 1A). After SPS stimulation, the amygdala neural cells became smaller, and the chromatin concentrated around the nuclear membrane. The nuclear membrane was damaged, especially in the SPS 7-day rats (Fig. 1B). Our previous study found there were different sizes of bubbles in the cytoplasm in the hippocampal cells, but we did not observe presence of bubbles in the cells from the amygdala.

Cell nuclear changes. DAPI is used to stain nuclei so that DNA can be visualized. We found differences in 4',6-diamidino-2-phenylindole-stained nuclear fluorescence staining intensities among the control and SPS groups (Fig. 2A-E). SPS increased staining intensity in cell nuclei, and reached a peak after 7 days $(\mathrm{P}<0.05$; Fig. $2 \mathrm{H})$. The magnification images showed normal cellular nuclei (Fig. 2F, arrowhead) and abnormal cell nuclei (Fig. 2G, arrow). Nuclei of normal cells showed low-intensity staining, but the abnormal cells were smaller and had higher intensity staining because of condensed chromatin and nuclear pycnosis that were visualized by electron microscopy (Fig. 1A and B). 
Table II. Primers for reverse transcription-quantitative polymerase chain reaction.

\begin{tabular}{lcc}
\hline Name size (bp) & Primer & Product size (bp) \\
\hline Cyclin D1 & & \\
Sense & 5'-GAGACCATTCCCCTGACTGC-3' & 79 \\
Antisense & 5'-CCATTTGCAGCAACTCCTCG-3' \\
CDK4 & & 182 \\
Sense & 5'-GGAGGCCTTTGAACATCCCA-3' \\
Antisense & 5'-ACTGGCGCATCAGATCCTTA-3' \\
GAPDH & & \\
Sense & 5'-ACTTTGGCATCGTGGAAGGG-3' \\
Antisense & 5'-ACTTGGCAGGTTTCTCCAGG-3' \\
\hline
\end{tabular}

CDK4, cyclin dependent kinase 4.

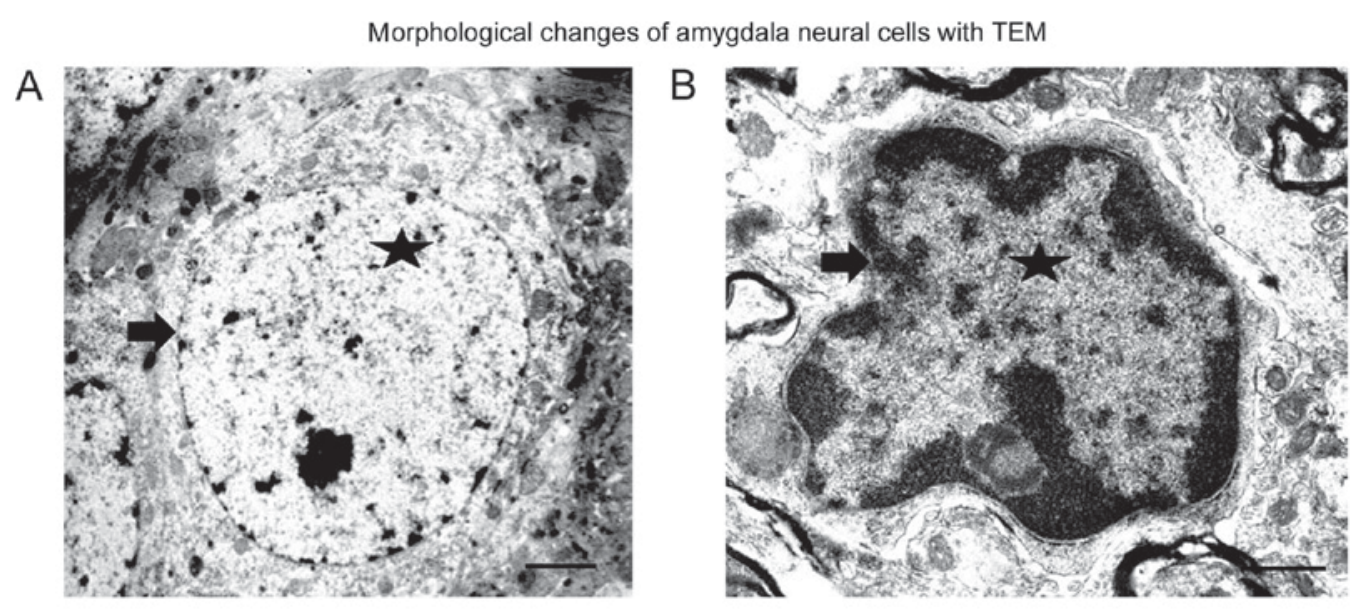

Figure 1. Transmission electron microcopy (TEM) images of amygdala neural cells. Nucleus is indicated by the star; nuclear membrane with arrow head. In the amygdala cells of (A) control group, the nuclear structure is clear and the nuclear membrane is complete, but in the amygdala cells of (B) single-prolonged stress rats, abnormal morphology in the nucleus and nuclear membrane was observed. Scale bar, $300 \mathrm{~nm}$.

Detection of CDK4 using immunofluorescence. Amygdala cell CDK4 was labeled with Cy3 fluorescent staining, and the CDK4 positive cells had red fluorescence. Apoptosis in the amygdala was measured by co-staining of CDK4 (red) and DAPI (blue) after SPS stimulation. With high-intensity CDK4 staining of the cytoplasm, the DAPI staining of the CDK4-immunoreactivity (ir)-positive cells was barely visible, indicating that CDK4 was only expressed in the non-apoptotic cells, with none in apoptotic cells. As shown in Fig. 3A, CDK4-ir was relatively weak, and there were few positive cells in the control group. At 1 and 4 days after SPS exposure, CDK4-ir did not show significant change in the amygdala cells. However, SPS significantly increased CDK4 expression at 7 days after SPS (Fig. 3D, n=5, $\mathrm{F}=32.56, \mathrm{P}<0.05$ ) and 14 days after SPS (Fig. 3E, $\mathrm{n}=5, \mathrm{~F}=16.91$, $\mathrm{P}<0.05)$. The differences in fluorescence intensity between the SPS group at 7 and 14 days, and the control group were statistically significant $(\mathrm{P}<0.05$; Fig. $3 \mathrm{~F})$.

Detection of cyclin D1 and CDK4 expression with western blotting. As shown in Fig. 4A, cyclin D1 protein levels were increased in the amygdala cells, compared with controls at 1 , 4 and 7 days after SPS stimulation. Interestingly, cyclin D1 protein levels increased with the duration of SPS stimulation. Cyclin D1 significantly increased at 4 days $(n=5, F=14.32$, $\mathrm{P}<0.05)$ and reached a peak at 7 days $(\mathrm{n}=5, \mathrm{~F}=30.21, \mathrm{P}<0.05)$, and maintained a relatively high level at 14 days $(n=5, F=23.67$, $\mathrm{P}<0.05$ ) in comparison with control group. Similar results were observed for CDK4 protein level in the SPS group. The comparison at 4 days $(\mathrm{n}=5, \mathrm{~F}=17.45, \mathrm{P}<0.05), 7$ days $(\mathrm{n}=5$, $\mathrm{F}=18.82, \mathrm{P}<0.05)$, and 14 days $(\mathrm{n}=5, \mathrm{~F}=19.38, \mathrm{P}<0.05)$ after SPS and controls was statistically significant ( $\mathrm{P}<0.05$; Fig. 4B).

Detection of cyclin D1 mRNA and CDK4 mRNA expression using $q P C R$. qPCR results showed that after SPS stimulation, at 1, 4, 7 and 14 days, the expression of cyclin D1 mRNA and CDK4 mRNA in amygdala neurons increased more than in the control group. This increasing trend of CDK4 mRNA was observed with the extension of stimulation time, peaking at 7 days $(\mathrm{n}=5, \mathrm{~F}=13.28, \mathrm{P}<0.05)$ and maintaining a high level at 14 days $(\mathrm{n}=5, \mathrm{~F}=19.36, \mathrm{P}<0.05)$. The relative expression of cyclin D1 mRNA in SPS rats at 7 days $(n=5, F=25.39, P<0.05)$ and 14 days $(n=5, F=19.68, P<0.05)$ after initial stimulation was statistically significant compared with control group ( $\mathrm{P}<0.05$; Fig. 5). 

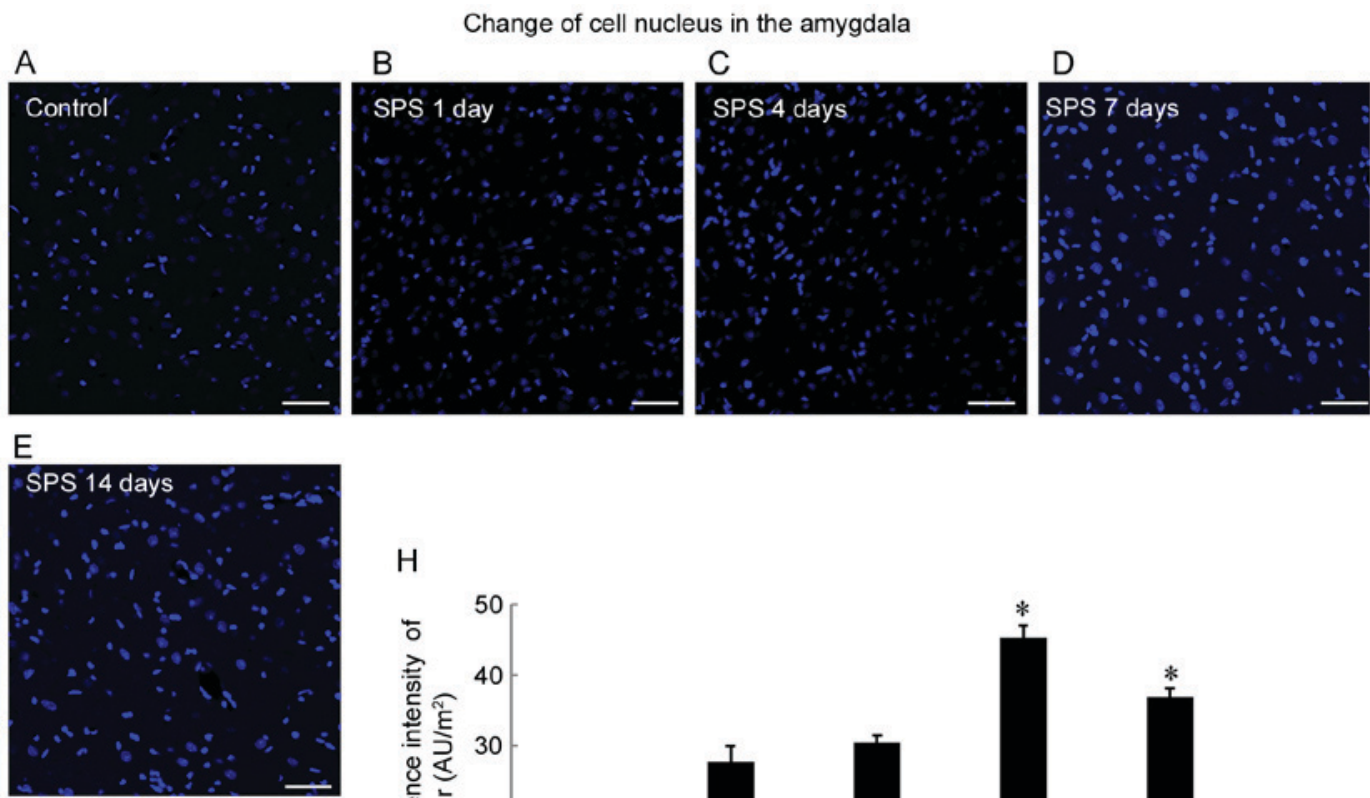

$\mathrm{F}$

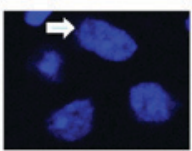

G
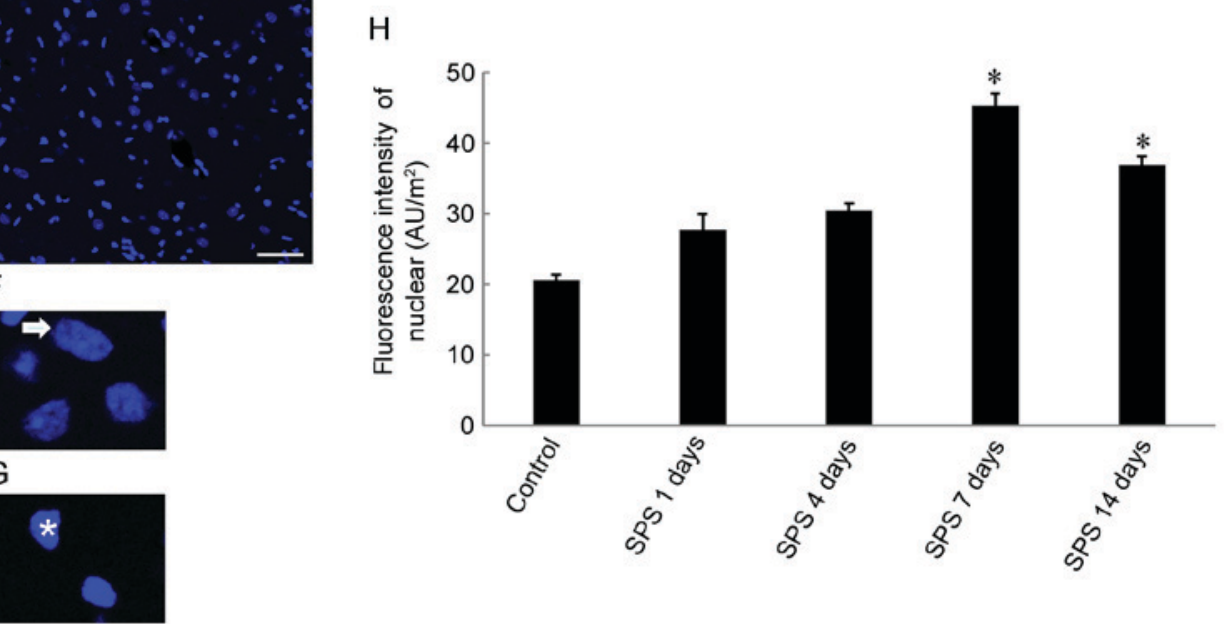

Figure 2. Visualized images showing intensity of DAPI stain from (A) control group, (B) single-prolonged stress (SPS) 1-day group, (C) SPS 4-day group, (D) SPS 7-day group, and (E) SPS-14 day group. The magnified image shows (F) nuclei of normal cell and (G) abnormal cell. (H) Show fluorescence intensity of cell nucleus $\left(\mathrm{AU} / \mu \mathrm{m}^{2}\right)$. ${ }^{*} \mathrm{P}<0.05$ vs. control group; scale bar, $50 \mu \mathrm{m}$.

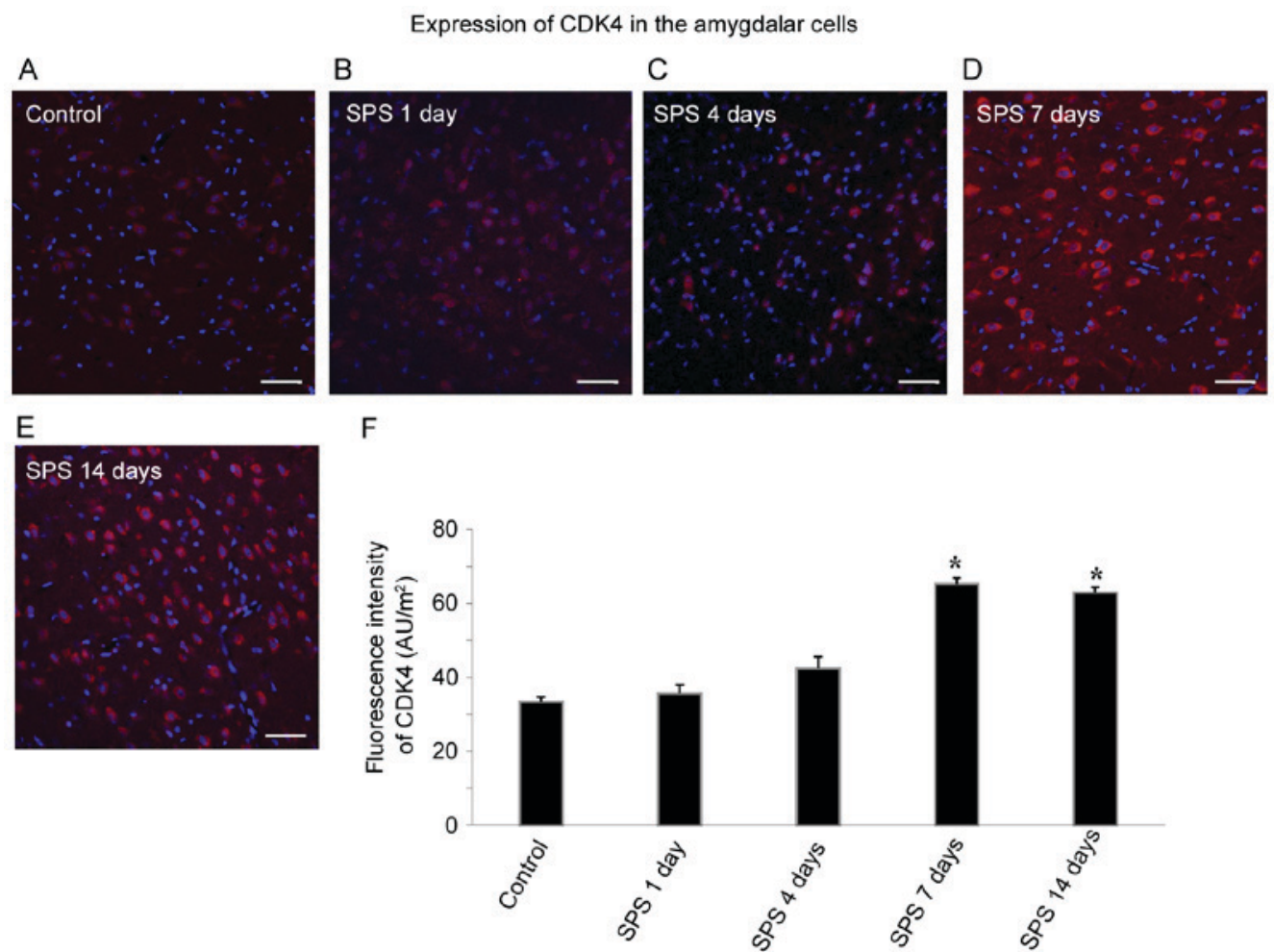

Figure 3. Expression of cyclin-dependent kinase 4 (CDK4) in amygdala neurons detected by Cy3 fluorescence labeling. CDK4-immunoreactivity (CDK4-ir) in the amygdalae of single-prolonged stress (SPS) rats in different groups (red): (A) Control group, (B) SPS 1-day group, (C) SPS 4-day group, (D) SPS 7-day group and (E) SPS 14-day group $\left(\mathrm{AU} / \mu \mathrm{m}^{2}\right)$. (F) ${ }^{*} \mathrm{P}<0.05$ vs. control group; scale bar, $50 \mu \mathrm{m}$. 
Detection of CDK4 and cyclin D1 expression with western blotting
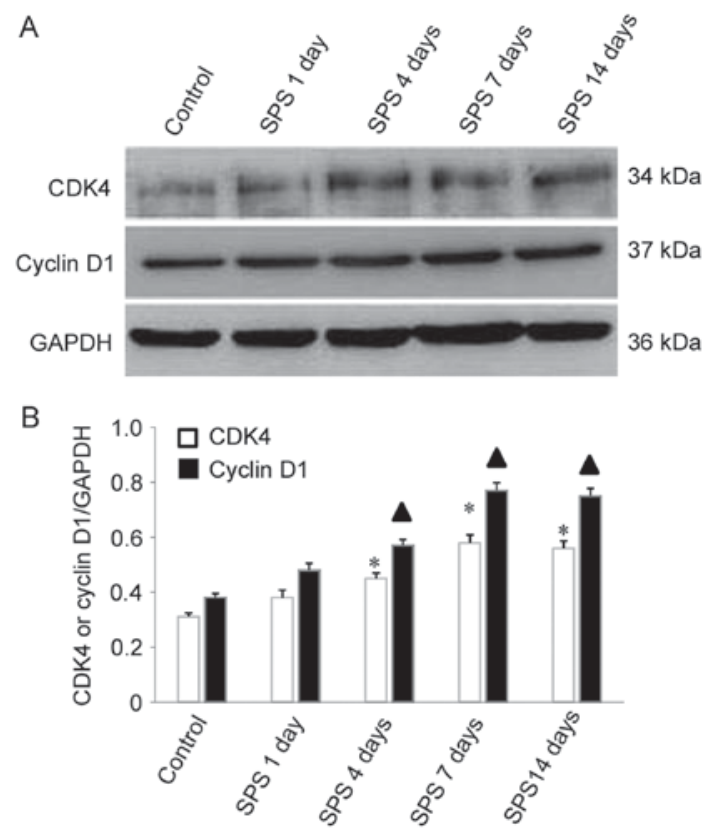

Figure 4. Western blot of cyclin-dependent kinase 4 (CDK4) and cyclin D1 expression in the amygdalae of SPS and control rats. (A) CDK4 and cyclin D1 protein expression. (B) Results from quantitative analysis based on Western blots. An increase in CDK4 and cyclin D1 protein expression is observed in single-prolonged stress (SPS) rats. " and $\Delta \mathrm{P}<0.05$ vs. control group.

Detection of Cyclin D1mRNA and CDK4 mRNA expression using real time PCR

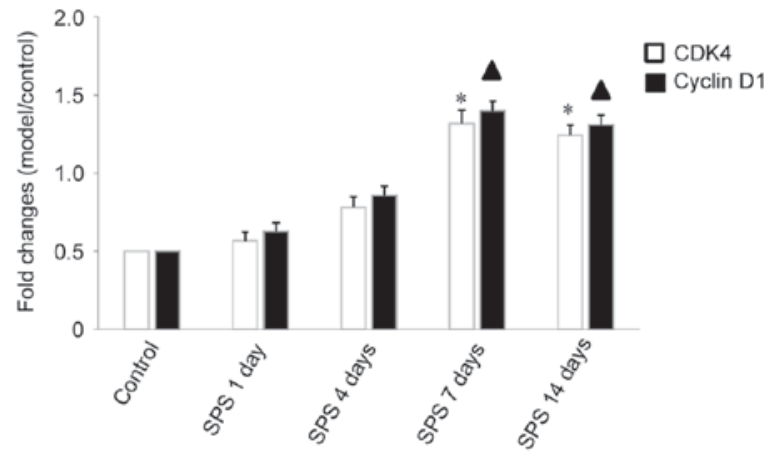

Figure 5. Quantitative polymerase chain reaction (PCR) of cyclin D1 mRNA and cyclin-dependent kinase 4 (CDK4) mRNA in the amygdalae of single-prolonged stress (SPS) and control rats. Results are from quantitative analysis of cyclin D1 mRNA and CDK4 mRNA expression. Cyclin D1 mRNA and CDK4 mRNA expression in the amygdalae of SPS rats was higher than that in control rats. ${ }^{*}$ and $\mathbf{\Delta}: \mathrm{P}<0.05$ vs. the control group.

\section{Discussion}

PTSD is an anxiety-related neurological disorder and mental illness caused by severe stimulation by stressors. About $10 \%$ of people will experience PTSD after exposure to a serious stressful event (37). One of the features of PTSD is an exaggerated fear response that does not resolve with the passage of time after the stimulating event, even in the absence of continuous stimuli. PTSD patients react strongly to fearful situations and may experience a strong sense of helplessness (38). Amygdalae are involved in the regulation of emotion and play a key role in fear memory. Magnetic resource imaging (MRI) studies reveal significant volume reductions in the amygdalae of adult patients with PTSD $(39,40)$. Our previous study noted a higher apoptosis rate in the amygdalae of SPS rats with TdT-mediated dUTP nick-end labeling (TUNEL)-staining and double-labeled flow cytometry methods. Alterations in apoptosis-related proteins (Bcl-2 and Bax) also occurred in the amygdala of the SPS rats (23). These studies suggest that abnormal structure and function in the amygdala is involved in PTSD.

Our previous studies used TUNEL and flow cytometry to show that SPS induced enhanced apoptosis in cells in the amygdala, hippocampus, and medial prefrontal cortex $(23,41,42)$. In this experiment, we observed through TEM that apoptosis occurred after SPS stimulation. Specifically, it was represented by a declining presence of cell bodies, cytoplasm concentration, and a frontier set of the chromatin. DAPI fluorescence was employed to detect nuclear pycnosis. After SPS stimulation, the fluorescence of cell nuclei in the amygdala increased gradually as a consequence of the pycnotic changes. These results prove that SPS increased neural death in the amygdala, which may contribute to the pathogenesis of PTSD. Functional changes of amygdala cells caused by PTSD may be the neural structural underpinning of PTSD symptoms.

Research has shown that in damaged axons, certain regulatory factors of the cell cycle, especially cyclin Dl and CDK4, are highly expressed $(43,44)$, and may play a significant role in the induction of cell apoptosis after central lesions.

The location of CDK4 expression in neurons of the amygdala of PTSD-like rats was observed using immunofluorescence, and quantitative analysis of cyclin D1 and CDK4 was performed by western blotting and qPCR. Cyclin D1 and CDK4 expression was gradually upregulated after SPS stimulation, peaking after $7 \mathrm{~d}$. This high expression of cyclin D1 and CDK4 suggests that PTSD may activate the neural cell cycle, and the high expression of cyclin D1 and CDK4 fails to play a regulating and controlling role in the cycle. Such unusually high activation of the cell cycle may be one of the main causes of neural cell apoptosis. The following reasons are possible: Mature neural cells are completely differentiated, and will not enter the cell cycle in normal and non-pathological conditions, and the expression time and trend of cyclin Dl and CDK4 matches that of the neural cell apoptosis. Using immunofluorescence, we discovered that CDK4 only exists in non-apoptotic cytoplasm, suggesting that it may play a role in the cell apoptosis process, as a significant factor influencing or inducing cell apoptosis.

We discovered that amygdala neurons went into apoptosis in PTSD-like SPS rats and consider that the high expression of cyclin Dl and CDK4 in amygdala neurons may accelerate the apoptosis of these cells. Possible mechanisms include the following factors: (1) Due to SPS stimulation, cyclin Dl and CDK4 are highly expressed in neural cells. The proliferation signal sent by cyclin Dl and CDK4 to cells is contradictory and incorrect, which may promote the expression of another apoptotic factor or directly triggers apoptosis. (2) Following SPS stimulation, high expression of cyclin Dl activates the cell cycle, but a lack of necessary enzymes for processing from the G1 to $\mathrm{S}$ phase 1 occurs, which may result in slowing of the cell cycle and cause apoptosis. (3) After SPS simulation, the CDK4/cyclin D1-retinoblastoma protein (pRB)-E2F signal transduction pathway is activated, and phosphorylated $\mathrm{pRB}$ is 
highly expressed, releasing free E2F1, which may be related to cell apoptosis (45).

In our study, SPS is used to establish the PTSD. Evidences from behavioral and neuroendocrinological studies examined that 7 days exposed to SPS is needed to develop PTSD. In the present study, we focus on the expression of CDK4 and cyclin D1 at 1, 4, 7 and 14 days after SPS exposure to explore different change of both factors during PTSD development (including acute period: 1 day and 4 days after SPS exposure). We did not find significant change in CDK 4 and cyclin D1 in the amygdala.

In conclusion, the experimental results show that changes in the expression of cyclin D1 and CDK4 may accelerate apoptosis of amygdala neurons in PTSD-like rats. Treatments interfering with the protein expression of the cell cycle, administered soon after trauma-induced stress, may lower the apoptosis of neural cells. This new hypothesis leads to a potential strategy for treatment of PTSD.

\section{Acknowledgements}

The present study was supported by the National Natural Science Foundation of China (no. 31140060), the Science and Technology Planning Project of Shenyang (no. F16-205-1-35), and the Natural Science Foundation of Liaoning Province, China (no. .201602274).

\section{References}

1. Bremner JD: The relationship between cognitive and brain changes in posttraumatic stress disorder. Ann N Y Acad Sci 1071: 80-86, 2006

2. Francati V, Vermetten E and Bremner JD: Functional neuroimaging studies in posttraumatic stress disorder: Review of current methods and findings. Depress Anxiety 24: 202-218, 2007.

3. Brown VM, LaBar KS, Haswell CC, Gold AL; Mid-Atlantic MIRECC Workgroup, McCarthy G and Morey RA: Altered resting-state functional connectivity of basolateral and centromedial amygdala complexes in posttraumatic stress disorder. Neuropsychopharmacology 39: 351-359, 2014.

4. Bahraini NH, Breshears RE, Hernández TD, Schneider AL, Forster JE and Brenner LA: Traumatic brain injury and posttraumatic stress disorder. Psychiatr Clin North Am 37: 55-75, 2014.

5. Klumpers F, Morgan B, Terburg D, Stein DJ and van Honk J: Impaired acquisition of classically conditioned fear-potentiated startle reflexes in humans with focal bilateral basolateral amygdala damage. Soc Cogn Affect Neurosci 10: 1161-1168, 2015.

6. Harding AJ, Stimson E, Henderson JM and Halliday GM: Clinical correlates of selective pathology in the amygdala of patients with Parkinson's disease. Brain 11: 2431-2445, 2002.

7. Vyas A, Mitra R, Shankaranarayana Rao BS and Chattarji S: Chronic stress induces contrasting patterns of dendritic remodeling in hippocampal and amygdaloid neurons. J Neurosci 22: 6810-6818, 2002.

8. Miller MM and McEwen BS: Establishing an agenda for translational research on PTSD. Ann N Y Acad Sci 1071: 294-312, 2006

9. Sarro EC, Sullivan RM and Barr G: Unpredictable neonatal stress enhances adult anxiety and alters amygdala gene expression related to serotonin and GABA. Neuroscience 258: 147-161, 2014.

10. El Khoury-Malhame M, Reynaud E, Soriano A, Michael K, Salgado-Pineda P, Zendjidjian X, Gellato C, Eric F, Lefebvre MN and Rouby F, et al: Amygdala activity correlates with attentional bias in PTSD. Neuropsychologia 49: 1969-1973, 2011.

11. Hayes JP, Hayes SM and Mikedis AM: Quantitative meta-analysis of neural activity in posttraumatic stress disorder. Biol Mood Anxiety Disord 2: 9, 2012.

12. Kühn S and Gallinat J: Gray matter correlates of posttraumatic stress disorder: A quantitative meta-analysis. Biol Psychiatry 73 70-74, 2013.
13. Morey RA, Gold AL, LaBar KS, Beall SK, Brown VM, Haswell CC, Nasser JD, Wagner HR and McCarthy G; Mid-Atlantic MIRECC Workgroup: Amygdala volume changes in posttraumatic stress disorder in a large case-controlled veterans group. Arch Gen Psychiatry 69: 1169-1178, 2012.

14. Brenner LA: Neuropsychological and neuroimaging findings in traumatic brain injury and post-traumatic stress disorder. Dialogues Clin Neurosci 13: 311-323, 2011.

15. Fu XL and Gao DS: Endoplasmic reticulum proteins quality control and the unfolded protein response: The regulative mechanism of organisms against stress injuries. Biofactors 40: 569-585, 2014.

16. Liu H, Wang HT, Han F and Shi YX: Activity of 5-HT1A receptor is involved in neuronal apoptosis of the amygdala in a rat model of post-traumatic stress disorder. Mol Med Rep 4: 291-295, 2011.

17. Wimalawansa SJ: Mechanisms of developing post-traumatic stress disorder: New targets for drug development and other potential interventions. CNS Neurol Disord Drug Targets 13: 807-816, 2014

18. Kohda K, Harada K, Kato K, Hoshino A, Motohashi J, Yamaji T, Morinobu S, Matsuoka N and Kato N: Glucocorticoid receptor activation is involved in producing abnormal phenotypes of single prolonged stress rats: A putative post-traumatic stress disorder model. Neuroscience 148: 22-33, 2007.

19. Li XM, Han F, Liu DJ and Shi YX: Single-prolonged stress induced mitochondrial-dependent apoptosis in hippocampus in the rat model of post-traumatic stress disorder. J Chem Neuroanat 40: 248-255, 2010.

20. Liu H, Li H, Xu A, Kan Q and Liu B: Role of phosphorylated ERK in amygdale neuronal apoptosis in single-prolonged stress rats. Mol Med Rep 3: 1059-1063, 2010.

21. Xiao B, Han F and Shi YX: Dysfunction of Ca2+/CaM kinase IIalpha cascades in the amygdala in post-traumatic stress disorder. Int J Mol Med 24: 795-799, 2009.

22. Xiao B, Yu B, Wang HT, Han F and Shi YX: Single-prolonged stress induces apoptosis by activating cytochrome C/caspase-9 pathway in a rat model of post-traumatic stress disorder. Cell Mol Neurobiol 31: 37-43, 2011.

23. Ding J, Han F and Shi Y: Single-prolonged stress induces apoptosis in the amygdala in a rat model of post-traumatic stress disorder. J Psychiatr Res 44: 48-55, 2010.

24. Milewska M, Grabiec K and Grzelkowska-Kowalczyk K: Interactions of proliferation and differentiation signaling pathways in myogenesis. Postepy Hig Med Dosw (Online) 68: 516-526, 2014.

25. Moschovi M, Alexiou GA, Patereli A, Siozos G, Sfakianos G, Prodromou N and Stefanaki K: Immunohistochemical expression of cell-cycle regulators in pediatric embryonal brain tumors. J Neurooncol 109: 529-534, 2012.

26. Buendía-Monreal M, Rentería-Canett I, Guerrero-Andrade O, Bravo-Alberto CE, Martínez-Castilla LP, García E and Vázquez-Ramos JM: The family of maize D-type cyclins: Genomic organization, phylogeny and expression patterns. Physiol Plant 143: 297-308, 2011

27. Casimiro MC, Velasco-Velázquez M, Aguirre-Alvarado C and Pestell RG: Overview of cyclins D1 function in cancer and the CDK inhibitor landscape: Past and present. Expert Opin Invest Drugs 23: 295-304, 2014.

28. Lee Y, Dominy JE, Choi YJ, Jurczak M, Tolliday N, Camporez JP, Chim H, Lim JH, Ruan HB, Yang X, et al: Cyclin D1-Cdk4 controls glucose metabolism independently of cell cycle progression. Nature 510: 547-551, 2014.

29. Kabadi SV and Faden AI: Selective CDK inhibitors: Promising candidates for future clinical traumatic brain injury trials. Neural Regen Res 9: 1578-1580, 2014.

30. Wu J, Stoica BA, Dinizo M, Pajoohesh-Ganji A, Piao C and Faden AI: Delayed cell cycle pathway modulation facilitates recovery after spinal cord injury. Cell Cycle 11: 1782-1795, 2012.

31. Folch J, Junyent F, Verdaguer E, Auladell C, Pizarro JG, Beas-Zarate $\mathrm{C}$, Pallàs $\mathrm{M}$ and Camins $\mathrm{A}$ : Role of cell cycle re-entry in neurons: A common apoptotic mechanism of neuronal cell death. Neurotox Res 22: 195-207, 2012.

32. Wang W, Bu B, Xie M, Zhang M, Yu Z and Tao D: Neural cell cycle dysregulation and central nervous system diseases. Prog Neurobiol 89: 1-17, 2009.

33. Pei L, Zhang Y, Zhang Y, Chu X, Zhang J, Wang R, Liu M, Zhu X and $\mathrm{Yu}$ W: Peroxisome proliferator-activated receptor gamma promotes neuroprotection by modulating cyclin D1 expression after focal cerebral ischemia. Can J Physiol Pharmacol 88: 716-723, 2010. 
34. Lee CH, Yoo KY, Choi JH, Park OK, Hwang IK, Choi SY, Kim DH and Won MH: Cyclin D1 immunoreactivity changes in CA1 pyramidal neurons and dentate granule cells in the gerbil hippocampus after transient forebrain ischemia. Neurol Res 33 93-100, 2011.

35. Liberzon I, Krstov M and Young EA: Stress-restress: Effects on ACTH and fast feedback. Psychoneuroendocrinology 22: 443-453, 1997.

36. Paxinos $\mathrm{G}$ and Watson $\mathrm{C}$ : The rat brain in stereotaxic coordinates. 4th edition. Academic Press, San Diego. CA, 1998.

37. Koch SB, van Zuiden M, Nawijn L, Frijling JL, Veltman DJ and Olff M: Intranasal oxytocin as strategy for medication-enhanced psychotherapy of PTSD: Salience processing and fear inhibition processes. Psychoneuroendocrinology 40: 242-256, 2014.

38. Amos T, Stein DJ and Ipser JC: Pharmacological interventions for preventing post-traumatic stress disorder (PTSD). Cochrane Database Syst Rev 7: CD006239, 2014.

39. Karl A, Schaefer M, Malta LS, Dörfel D, Rohleder N and Werner A: A meta-analysis of structural brain abnormalities in PTSD. Neurosci Biobehav Rev 30: 1004-1031, 2006.

40. Driessen M, Beblo T, Mertens M, Piefke M, Rullkoetter N, Silva-Saavedra A, Reddemann L, Rau H, Markowitsch HJ, Wulff $\mathrm{H}$, et al: Posttraumatic stress disorder and fMRI activation patterns of traumatic memory in patients with borderline personality disorder. Biol Psychiatry 55: 603-611, 2004.
41. Li X, Han F, Liu D and Shi Y: Changes of Bax, Bcl-2 and apoptosis in hippocampus in the rat model of post-traumatic stress disorder. Neurol Res 32: 579-586, 2010.

42. Li Y, Han F and Shi Y: Increased neuronal apoptosis in medial prefrontal cortex is accompanied with changes of $\mathrm{Bcl}-2$ and Bax in a rat model of post-traumatic stress disorder. J Mol Neurosci 51: 127-137, 2013.

43. Nobs L, Nestel S, Kulik A, Nitsch C and Atanasoski S: Cyclin D1 is required for proliferation of Olig2-expressing progenitor cells in the injured cerebral cortex. Glia 61: 1443-1455, 2013.

44. Kabadi SV, Stoica BA, Loane DJ, Byrnes KR, Hanscom M, Cabatbat RM, Tan MT and Faden AI: Cyclin D1 gene ablation confers neuroprotection in traumatic brain injury. J Neurotrauma 29: 813-827, 2012.

45. Carnevale J, Palander O, Seifried LA and Dick FA: DNA damage signals through differentially modified E2F1 molecules to induce apoptosis. Mol Cell Biol 32: 900-912, 2012. 